\title{
AN AUTOMATIC COLLISION AVOIDANCE ALGORITHM FOR MULTIPLE MARINE SURFACE VEHICLES
}

\author{
RAMDANE HEDJAR $^{a, *}$, MESSAOUD BOUNKHEL $^{b}$ \\ ${ }^{a}$ Department of Computer Engineering \\ King Saud University, PO Box 5117, Riyadh 11543, Saudi Arabia \\ e-mail: hedjar@ksu.edu.sa \\ ${ }^{b}$ Department of Mathematics \\ King Saud University, PO Box 2455, Riyadh 11451, Saudi Arabia \\ e-mail: bounkhel@ksu.edu.sa
}

\begin{abstract}
In recent years, unmanned surface vehicles have been widely used in various applications from military to civil domains. Seaports are crowded and ship accidents have increased. Thus, collision accidents occur frequently mainly due to human errors even though international regulations for preventing collisions at seas (COLREGs) have been established. In this paper, we propose a real-time obstacle avoidance algorithm for multiple autonomous surface vehicles based on constrained convex optimization. The proposed method is simple and fast in its implementation, and the solution converges to the optimal decision. The algorithm is combined with the PD-feedback linearization controller to track the generated path and to reach the target safely. Forces and azimuth angles are efficiently distributed using a control allocation technique. To show the effectiveness of the proposed collision-free path-planning algorithm, numerical simulations are performed.
\end{abstract}

Keywords: unmanned surface vehicles, obstacle avoidance, control allocation, constrained convex optimization.

\section{Introduction}

In the early $1970 \mathrm{~s}$, the International Maritime Organization (IMO) published international regulations for preventing collisions at sea (COLREGs). They outlined procedures that regulate traffic of vessels and reduce traffic-related accidents at sea which are mainly due to human errors. In 2005, the IMO advocated on-board integration of sensors, digital information processing, and their management in order to increase the safety and reduce marine accidents, which will also reduce financial losses that affect the marine sector. During the last decade, marine traffic has increased and currently ranges from civil applications (oil and gas exploration, transportation, etc.) to military applications (surveillance, mine detection, etc.). Therefore, the level of autonomous navigation of vessels should be increased and automatic obstacles avoidance should also be included in navigation systems, while respecting maritime traffic rules (Campbell et al., 2002). Consequently, autonomous navigation of surface vehicles, in the presence of other

* Corresponding author maritime traffic, will increase the safety, reliability and reduce the occurrence of vessels collisions.

Vessel collision avoidance becomes an important area in the maritime science. Many researchers and experts investigated various strategies for collision avoidance of ships at sea. Most of the proposed solutions are based on metaheuristic techniques (Statheros et al., 2008) or stochastic search algorithms (Kim et al., 2017). This is due to the fact that a multi-objective optimization problem under various constraints is encountered during the navigation. Perera et al. (2011) proposed a fuzzy-logic-based decision-making system for collision avoidance in oceanic navigation. Liu and Shi (2005) combined the fuzzy logic approach with neural networks to quantify collision avoidance decisions.

Most of these developed algorithms obey the rules of the road (COLREGs) (Benjamin et al., 2006; Statheros et al., 2008). Kuwata et al. (2014) utilized the velocity obstacle approach to develop an autonomous motion-planning algorithm for unmanned surface vehicles (USVs). A multi-objective optimization algorithm was 
adopted by $\mathrm{Xu}$ et al. (2014) to search for an optimal collision avoidance strategy. The artificial potential field method is proposed for multi-ship collision avoidance by Wang et al. (2017). An ant colony optimization approach was used by Lazarowska (2015) to generate a safe path of a ship navigating in a dynamic environment. The proposed solution can be used in decision support systems on board to generate a safe trajectory.

Erol et al. (2018) used a neuro-fuzzy approach and genetically optimized fuzzy classifiers to analyze marine accidents occurring inside narrow, shallow and busy waterways. The modified Wolf Pack algorithm is proposed by Zhang et al. (2017) to overcome the slow convergence rate and to improve the accuracy of the algorithm. The enhanced algorithm was applied for safety path planning of underwater vehicles. It should be noted that most of previous techniques are based on metaheuristic methods (nature-inspired approaches) that are approximate, non-deterministic, and the optimization problem must be solved on-line with computational complexity (Kvasov and Mukhametzhanov, 2018).

The algorithm described in this work addresses mainly the hazard collision avoidance for stationary and moving obstacles for unmanned surface vehicles operating alongside with other USVs. The algorithm is able to generate a safe path on-line by solving a constrained convex optimization problem. In practice, human operators neglect the COLREGs and this negligence is a cause of many maritime traffic accidents. Statheros et al. (2008) mentioned that $56 \%$ of ship accidents were mainly due to the human navigators that had not obeyed the rules of the road. Consequently, the proposed algorithm can be used in the second level as an emergency obstacle avoidance scheme while the algorithm that obeys the rules of the road (COLREGs) can be put in the first level.

In this work, an online path-planning algorithm for multiple USVs exploits the solution of a quadratic optimization problem with inequality constraints. Therefore, the main contribution of this work lies in the conversion of the navigation problem of USVs to a constrained quadratic (convex optimization) problem where the optimal safe direction is obtained explicitly. In this structure, each USV plans its own path based on the presence of static or moving obstacles. Therefore, each ship considers all other ships in the vicinity as obstacles.

The advantage of the proposed method over metaheuristic methods (Liu and Shi, 2005; Perera et al., 2011) is that it needs less processing time to reach an optimal decision. On the other hand, compared with the potential field method (Wang et al., 2017), the proposed solution is obtained by solving a convex optimization problem where the solution converges to a global optimum. Further, a control allocation approach is employed to allow efficient distribution of the control tasks over actuators.

The paper is organized as follows. Section 2 establishes the mathematical model of multiple marine surface vehicles. A state feedback linearization control approach combined with an allocation control scheme is described in this section. The problem formulation of the emergency obstacle avoidance algorithm is presented in Section 3. To assess the tracking performances of the proposed algorithm, numerical simulations are performed and the results are shown in Section 4. Section 5 contains some concluding remarks and summarizes the presented approach.

\section{Modelling and control of multiple surface vessels}

In this work we focus on a group of marine vessels, so the dynamic equation of a single marine craft is first considered and the dynamic model of a set of USVs is described in the next section. To this end, we consider the standard notation used in the marine field, where $\{I\}$ denotes the inertial coordinate frame, while $\{B\}$ the body-fixed coordinate frame attached to the centre of the vehicle. Thus, the dynamic model of the $i$-th ship in the body-fixed frame $B$ is given as (Fossen, 1994; Skjetne et al., 2005)

$$
\begin{aligned}
\dot{\eta}_{i} & =R\left(\psi_{i}\right) v_{i}, \\
M_{i} \dot{v}_{i}+C_{i}\left(v_{i}\right) v_{i}+D_{i}\left(v_{i}\right) v_{i} & =\tau_{i}+R\left(\psi_{i}\right)^{T} b_{i},
\end{aligned}
$$

where $R(\cdot) \in S O(3), S O(3)$ is the special orthogonal rotation matrix group, $M_{i}$ is the inertia matrix, $C_{i}\left(v_{i}\right)$ is the Coriolis-centripetal matrix, $D_{i}\left(v_{i}\right)$ is the nonlinear hydrodynamic damping matrix, and $b_{i}=\left[\begin{array}{lll}b_{1 i} & b_{2 i} & b_{3 i}\end{array}\right]^{T}$ corresponds to a slowly varying unknown disturbance due to external forces (wind and waves) acting on the $i$-th ship.

For surface vessels, a common used approximation from 6 DOFs to 3 DOFs is the horizontal plane model in surge, sway and yaw. Therefore, the variables of the model (1) in 3 DOFs form $\eta_{i}=\left[\begin{array}{lll}x_{i} & y_{i} & \psi_{i}\end{array}\right]^{T}$, the position-orientation vector. The orientation angle $\psi_{i}$ is the heading angle of the $i$-th ship in the inertial frame. Here $v_{i}=\left[\begin{array}{lll}u & \vartheta & r\end{array}\right]^{T}$ is composed of the linear velocities $(u, \vartheta)$ (or surge-sway) and the angular rate $r$ (or yaw). For small roll and pitch angles and no heave, the transformation matrix between the two frames can be approximated by

$$
R\left(\psi_{i}\right)=\left[\begin{array}{ccc}
\cos \left(\psi_{i}\right) & -\sin \left(\psi_{i}\right) & 0 \\
\sin \left(\psi_{i}\right) & \cos \left(\psi_{i}\right) & 0 \\
0 & 0 & 1
\end{array}\right] .
$$

The inertia matrix (including added mass) is given as 
follows (Fossen and Johansen, 2006; Skjetne et al., 2005):

$$
M_{i}=\left[\begin{array}{ccc}
m_{i}-X_{\dot{u}} & 0 & 0 \\
0 & m_{i}-Y_{\dot{\vartheta}} & m_{i} x_{g}-Y_{\dot{r}} \\
0 & m_{i} x_{g}-N_{\dot{\vartheta}} & I_{z}-N_{\dot{r}}
\end{array}\right]
$$

with the equality $Y_{\dot{v}}=N_{\dot{r}}$, the inertia matrix becomes symmetric $\left(M_{i}=M_{i}^{T}\right)$.

The Coriolis-centripetal matrix is

$$
C_{i}\left(v_{i}\right)=\left[\begin{array}{ccc}
0 & 0 & c_{13}\left(v_{i}\right) \\
0 & 0 & c_{23}\left(v_{i}\right) \\
-c_{13}\left(v_{i}\right) & c_{23}\left(v_{i}\right) & 0
\end{array}\right] \text {, }
$$

which is a skew-symmetric matrix $\left(C_{i}\left(v_{i}\right)=-C_{i}\left(v_{i}\right)^{T}\right)$ where $c_{13}\left(v_{i}\right)=-\left(m_{i}-Y_{\dot{v}}\right) \vartheta-\left(m_{i} x_{g}-Y_{\dot{r}}\right) r$, and $c_{23}\left(v_{i}\right)=\left(m_{i}-X_{\dot{u}}\right) u$. The nonlinear damping matrix is

$$
D_{i}\left(v_{i}\right)=\left[\begin{array}{ccc}
d_{11}\left(v_{i}\right) & 0 & 0 \\
0 & d_{22}\left(v_{i}\right) & d_{23}\left(v_{i}\right) \\
0 & d_{32}\left(v_{i}\right) & d_{33}\left(v_{i}\right)
\end{array}\right] \text {, }
$$

where $d_{11}\left(v_{i}\right)=-X_{u}-X_{|u| u}|u|-X_{u u u} u^{2} ; d_{22}\left(v_{i}\right)=$ $-Y_{\vartheta}-Y_{|\vartheta| \vartheta}|\vartheta|-Y_{|r|} \vartheta|r|, d_{23}\left(v_{i}\right)-Y_{r}-Y_{|\vartheta| r}|\theta|-$ $Y_{|r| r}|r| ; d_{32}\left(v_{i}\right)=-N_{\vartheta}-N_{|\vartheta| \vartheta}|\vartheta|-N_{|r| \vartheta}|r|$, and $d_{33}\left(v_{i}\right)=-N_{r}-N_{|\vartheta| r}|\vartheta|-N_{|r| r}|r|$.

Note that the coefficients $\left\{X_{(\cdot)}, Y_{(\cdot)}, N_{(\cdot)}\right\}$ denote the hydrodynamic parameters (Skjetne et al., 2005). The control vector $\tau_{i}=\left[\begin{array}{lll}\tau_{u} & \tau_{\vartheta} & \tau_{r}\end{array}\right]^{T}$ is composed of generalized forces and moments generated by the propeller revolutions and rudder angles.

2.1. Mathematical model of a set of USVs. Consider a set of $N$ marine crafts with the position given by $\eta_{i}$, inertia matrix $M_{i}$, velocity $v_{i}$ and so on for all variables given in (1) for $i=1,2, \ldots, N$. To express the dynamic equation of the set of marine crafts in compact form, we collect all vectors and matrices in new ones. Indeed, the Cartesian position and the orientation of a group of marine crafts at sea is defined by the vector $\eta=\left[\begin{array}{lll}\eta_{1}^{T} & \cdots & \eta_{N}^{T}\end{array}\right]$, their inertial matrix is $M=\operatorname{diag}\left(M_{1}, \cdots, M_{N}\right)$, the velocity vector is $v=$ $\left[\begin{array}{lll}v_{1}^{T} & \cdots & v_{N}^{T}\end{array}\right]^{T}$, and the extension can be done for all other vectors and matrices. Thus, the dynamic model of the unmanned surface vehicles becomes

$$
\begin{aligned}
\dot{\eta} & =R(\psi) v, \\
M \dot{v}+C(v) v+D(v) v & =\tau+R(\psi)^{T} b,
\end{aligned}
$$

where $\operatorname{dim}(\eta)=\operatorname{dim}(v)=\operatorname{dim}(\tau)=\operatorname{dim}(b)=3 N \times 1$. $\operatorname{dim}(M)=\operatorname{dim}(C)=\operatorname{dim}(D)=3 N \times N$;
2.2. State feedback linearization control. The feedback linearization is a transformation map used to transform a nonlinear system dynamics to a linear system dynamics in order to use various linear controllers available in the literature (Fossen and Johansen, 2006). In this work, the position tracking control is decoupled in the earth-fixed inertial frame $\{I\}$. Assume that each vehicle has its own desired trajectory $\eta_{\text {ref }_{i}}(t)$ (continuous and differentiable). The reference trajectory vector becomes $\eta_{\mathrm{ref}}=\left[\begin{array}{lll}\eta_{\mathrm{ref} 1}^{T} & \cdots & \eta_{\mathrm{refN}}^{T}\end{array}\right]$ and the tracking error as $e(t)=$ $\eta(t)-\eta_{\text {ref }}(t)$. The torque control vector is used to eliminate nonlinearities from the nonlinear model (Erol et al., 2018) and to ensure the asymptotic stability of the tracking error. Consider the following control vector (Isidori, 1989):

$$
\begin{aligned}
\tau= & -R(\psi)^{T} b+C(v) v+D(v) v \\
& +M R^{T}(\psi)\left(\ddot{\eta}_{\mathrm{ref}}-K_{d} \dot{e}-K_{p} e-\dot{R}(\psi) v .\right.
\end{aligned}
$$

Since $M>0$ and $R(\psi) \in S O(3)$, substituting (4) into (3), the closed-loop system in the inertial frame leads to

$$
\ddot{e}+K_{d} \dot{e}+K_{p} e=0,
$$

where the gains are chosen as $K_{d}=$ $2 \operatorname{diag}\left(\zeta_{1} \omega_{1}, \ldots, \zeta_{N} \omega_{N}\right)$ and $K_{p}=\operatorname{diag}\left(\omega_{1}^{2}, \ldots, \omega_{N}^{2}\right)$, where $0<\zeta_{i}<1$ is the damping ratio and $\omega_{i}>0$ is the natural frequency of the $i$-th vessel in the closed-loop. Therefore, the PD-feedback linearization controller ensures asymptotic convergence of the tracking error to zero. The natural frequency parameter is used to speed up the movement of the vessel in the absence of obstacles. However, in the presence of obstacles, this parameter is decreased in order to slow down the dynamics of vessels. The PD-feedback linearization controller is valid under the assumption that all parameters of the nonlinear model (3) are known. To overcome this issue, a parameter adaptation law can be combined with the previous PD-controller (Skjetne et al., 2005). In addition, in this work it is assumed that all vessels are equipped with measurement systems that provide a full state measurement.

2.3. Nonlinear constrained control allocation. In marine vessels, the generalized control forces $\tau \in \mathbb{R}^{3}$, are distributed throughout available actuators (thruster, rudders, etc.) as control inputs $\gamma \in \mathbb{R}^{p}$ and $\theta \in(-\pi, \pi)^{r}$, where $p$ denotes the number of actuators. The relation between the generalized forces and the control signals is nonlinear and it is given as follows (Fossen, 1994; Johansen and Fossen, 2013):

$$
\tau_{\mathrm{sat}}=T(\theta) K_{\mathrm{ac}} \gamma=T(\theta) f
$$

where $K_{\mathrm{ac}} \in \mathbb{R}^{p \times p}$ is a diagonal force coefficient matrix, $f=K_{\mathrm{ac}} \gamma \mathbb{R}=\left[\begin{array}{lll}f_{1} & f_{2} & f_{3}\end{array}\right]^{T}$ is the vector of 
forces developed by the actuators, $\theta=\left[\begin{array}{ll}\theta_{1} & \theta_{2}\end{array}\right]^{T}$ is the vector of the azimuth angles of the rotatable thruster and $T(\theta)) \in \mathbb{R}^{3 \times p}$ is the actuator configuration matrix. The configuration matrix $T(\theta)$ is made by of a set of column vectors $T_{i}(\theta) \in \mathbb{R}^{3}$ and it is defined for $p=3$ (3 actuators among which two are rotatable) (Fossen, 1994) as

$$
T_{i}(\theta)=\left[\begin{array}{c}
\cos \left(\theta_{i}\right) \\
\sin \left(\theta_{i}\right) \\
l_{x i} \sin \left(\theta_{i}\right)-l_{y i} \cos \left(\theta_{i}\right)
\end{array}\right],
$$

for an azimuth thruster $(i=1,2)$ and $T_{3}(\theta)=$ $\left[\begin{array}{lll}0 & 1 & l_{x 3}\end{array}\right]^{T}$ for the tunnel rudder.

The control allocation problem permits to calculate, for each control torque $\tau$, the corresponding optimal set of control inputs $\left(f_{i}, \theta_{i}\right)$. To this end, the following nonlinear constrained optimization problem is solved (Fossen, 1994):

$$
\min _{\gamma, \theta} J=\sum_{i=1}^{3} p_{i}\left|f_{i}\right|^{\frac{3}{2}}+\theta^{T} Q \theta,
$$

such that

$$
\begin{aligned}
& \tau_{\text {sat }}-T(\theta) f=0, \\
& f_{\min } \leq f \leq f_{\max }, \\
& \theta_{\min } \leq \theta \leq \theta_{\max },
\end{aligned}
$$

where $p_{i}>0(i=1,3)$ are positive weights for actuators forces, $Q \in \mathbb{R}^{2 \times 2}$ is a positive-definite weighting matrix for azimuth angles. Here $f_{\min } \leq f \leq f_{\max }$ is the feasible interval of the applied forces and $\theta_{\min } \leq \theta \leq \theta_{\max }$ is the feasible interval for azimuth angles (Fossen and Johansen, 2006).

Due to the presence of nonlinear constraints, the optimization problem (7) is non-convex and to reach an optimal solution, the solver needs a huge amount of computations and time processing. Fossen and Johansen (2006) have used iterative solutions based on linear programming methods to solve this kind of non-convex optimization problem.

\section{Problem formulation}

Consider $N$ unmanned surface vehicles, with arbitrary shapes navigating at sea. The marine vehicle is included inside a virtual disk with radius of $R_{i}, i=1,2, \ldots, N$ (Fig. 1). The safety distance of each vehicle is denoted by

$$
\delta_{i}=R_{i}-r_{i}
$$

where $r_{i}$ is the radius of the vehicle or the maximum length of the ship from its centre. The goal of this work is to simulate a swarm of marine crafts inside a given space $\Omega \in \mathbb{R}^{2}$ that are subject to static and/or dynamic obstacles. Hence, we define the configuration space of
USVs at time $t$ by the vector $q(t)=\left[q_{1} \ldots q_{N}\right] \in \mathbb{R}^{2 N}$ where $q_{i}(t)=\left[\begin{array}{ll}x_{i}(t) & y_{i}(t)\end{array}\right]^{T}$ is the Cartesian position of the $i$-th vehicle. The configuration is admissible when the motion of different USVs is safe without collision or overlap. This can be expressed mathematically as

$$
W=\left\{q(t) \in \mathbb{R}^{2 N}: \triangle_{i j}(q(t)) \geq 0, \forall i, j\right\},
$$

where $\triangle_{i j}(q)=\left\|q_{i}-q_{j}\right\|-\left(R_{i}+R_{j}\right)$ denotes the distance between disks $i$ and $j$.

The safety navigation of USVs can be formulated as follows, starting from an admissible configuration at time $t, q(t) \in W$. To ensure a safe navigation of all vehicles at the next time $(t+h)$ for a small value of $h>0$, the next configuration should also be admissible, i.e., $q(t+h) \in W$. This implies that the constraint $\triangle_{i j}(q(t+h)) \geq 0$ should be satisfied. Using the first-order Taylor expansion, we deduce the constraint on the velocity vector as

$$
\triangle_{i j}(q(t+h))=\triangle_{i j}(q(t))+h \nabla \triangle_{i j}(q) \dot{q} \geq 0,
$$

where $\nabla \triangle_{i j}(q)$ is the gradient of the distance function.

This constraint will be used to generate the next configuration or the next reference position of USVs in order to avoid surrounding obstacles. In this regard, set the vector $V(q)$ to be the desired velocity of all USVs. The admissible velocities providing no collision during navigation of USVs are defined as

$$
\begin{aligned}
& C_{v}(q) \\
& =\left\{V(q) \in \mathbb{R}^{2 N}: \triangle_{i j}(q(t))+h \nabla \triangle_{i j} V(q) \geq 0\right\}
\end{aligned}
$$

Note that, in the absence of obstacles, the desired velocities are given by $V(q)=U(q)=q_{\mathrm{targ}}-q(t) \in$ $\Re^{2 N}$. In the presence of obstacles, the proposed algorithm

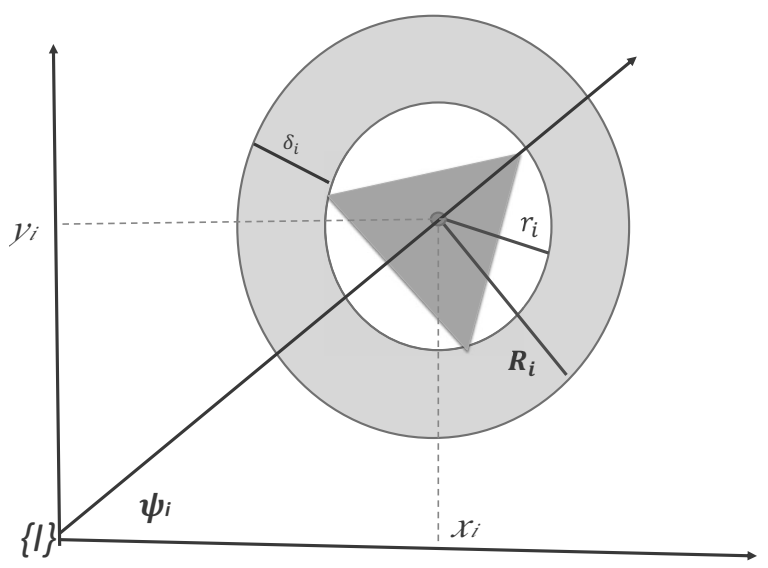

Fig. 1. Arbitrary shape of the marine vessel immersed in the disc. 
looks for optimal velocities to escape from different surrounding obstacles by solving the following convex constrained optimization problem:

$$
\min \|U(q)-V(q)\|^{2}
$$

subject to $V(q) \in C_{v}(q)$.

Consequently, the proposed scheme seeks for new directions $V(q)$ of USVs close to the desired direction $U(q)$ in order to bypass the surrounding obstacles. The desired position of the next configuration of marine vessels is then generated as $q_{\text {ref }}(t+h)=\left[\begin{array}{ll}x_{\text {ref }} & y_{\text {ref }}\end{array}\right]^{T}=$ $q(t)+h V(q)$ and the desired via-point posture position of the marine craft is

$$
\eta_{\text {ref }}(t)=\left[\begin{array}{lll}
x_{\text {ref }} & y_{\text {ref }} & \psi_{\text {ref }}
\end{array}\right]^{T},
$$

where

$$
\psi_{\mathrm{ref} i}=\tan ^{-1}\left(\frac{y_{\mathrm{ref} i}-y_{i}(t)}{x_{\mathrm{ref} i}-x_{i}(t)}\right) .
$$

Therefore, the control algorithm (4) with (12) can be used to compute the optimal torques to escape collisions with surrounding obstacles and consequently move the marine vessel safely to the target. The optimization problem (11) is a quadratic programming (QP) problem. Thus, many standard numerical algorithms are available to solve this kind of problem. Further, a combination of new coding approaches, and increased computing power may solve this convex optimization problem in milliseconds (Mattingley and Boyd, 2010)

\section{Simulation results}

To verify the effectiveness of the proposed obstacle avoidance algorithm applied to multiple unmanned surface vehicles, computer simulations were carried out using MATLAB. To this end, we adopted two different scenarios in our numerical simulations. The first is the case with a static obstacle, and the second with no static obstacle.

\section{Case 1. (A static obstacle)}

The first scenario that has been set up comprises three marine crafts where two are moving vehicles ( $\mathrm{craft}_{1}$ and $\left.\mathrm{craft}_{2}\right)$ while the third one $\left(\mathrm{craft}_{3}\right)$ is static. The marine surface vehicles are represented by triangle shapes immersed in discs (Fig. 11). The objective is to move craft $_{1}$ and craft $_{2}$ to their corresponding targets (Targ ${ }_{1}$ and $\operatorname{Targ}_{2}$ without colliding with the static obstacle and with each other. However, in the presence of obstacles, the vehicle utilizes the via-point reference trajectory induced from the collision-free path planning algorithm (11) and determines the adequate control vector to be applied to the $i$-th marine vehicle $(i=1,2)$, using the PD controller (4) with control allocation (7)). In the absence of obstacles, the marine surface vehicle uses the PD feedback
Algorithm 1. Real-time obstacle avoidance for the vessel $i$.

1. Initialization:

- control parameters: $h, \omega_{i}, \zeta_{i}$ and $q_{i}^{\mathrm{targ}}$

- mathematical model coefficients in Eqn. (1),

- $R_{i}, r_{i}, \delta_{i}, d_{\text {threshold }}, \varepsilon$,

- initial position: $q_{i}\left(t_{k}\right)=q_{i}(0)$,

- threshold distances: $\varepsilon_{i}, d_{C R}, \sigma$

2. While $\left\|q_{i}\left(t_{k}\right)-q_{i}^{\operatorname{targ}}\right\| \geq \varepsilon_{i}$ do

1. Measure the position $\eta_{i}\left(t_{k}\right)$.

2. Set the direction to the target: $U_{i}\left(t_{k}\right)=q_{i}^{\operatorname{targ}}-q_{i}\left(t_{k}\right)$.

3. Read sensors to detect obstacle: $d_{\text {obs }}$,

4. If $d_{\mathrm{obs}} \gg \infty$; No obstacles surroundings: $q_{\mathrm{ref} i} \longleftarrow$ $q_{i}\left(t_{k}\right)+h U_{i}\left(t_{k}\right)$ and go to step:7

5. If $\sigma \leq d_{\text {obs }} \leq d_{C R} ; q_{\text {ref }} \leftarrow$ use COLREGs for obstacles avoidance and go to Step 7

6. If $d_{\mathrm{obs}} \leq \sigma$ : Enable the emergency obstacles avoidance algorithm: solve $\min \left\|U_{i}(q)-V_{i}(q)\right\|^{2}$ subject to $V_{i}(q) \in C_{v}(q): \quad q_{\mathrm{ref} i} \leftarrow q_{i}\left(t_{k}\right)+$ $h V_{i}\left(q\left(t_{k}\right)\right)$,

7. Calculate $\eta_{\text {ref } i}$,

8. Compute the toque $\tau_{i}$ using Eqn. (4) with $e_{i}=$ $\eta_{i}(t)-\eta_{\mathrm{ref} i}$

9. Control allocation: Compute and apply $f_{i}$ and $\theta_{i}$.

3. End of the While loop.

linearization controller where the tracking error is the difference between the actual position of the USV and its desired position. MATLAB Optimization Toolbox is used to solve the non-convex optimization problem given in (7).

For simplicity, identical marine vehicles are taken in this work. The mathematical model is taken from the physical ship called Cyber-Ship (Skjetne et al., 2005), where its mass is $23.8 \mathrm{~kg}$ and its length is $1.255 \mathrm{~m}$. The parameters of the mathematical model (1) are shown in Table 1.

The control parameters used in the simulation for the gains $K_{p}$ and $K_{d}$ are $\zeta=1$ and for the natural frequency $\omega_{n}=4 \mathrm{rad} / \mathrm{s}$ in the absence of obstacles, while $\omega_{n}=0.8$ $\mathrm{rad} / \mathrm{s}$ in the presence of obstacles. Torque signals are saturated within $\tau_{\max }=2 \mathrm{Nm}$ and $\tau_{\min }=-2 \mathrm{Nm}$. The initial configuration is $q(0)=\left[\begin{array}{lll}q_{1}(0) & q_{2}(0) & q_{3}(0)\end{array}\right]^{T}$ where $q_{1}(0)=\left[\begin{array}{ll}0 & 0\end{array}\right]^{T}$ is the initial position of the 
Table 1. Mathematical model parameters.

\begin{tabular}{|c|c|c|c|}
\hline Parameter & value & Parameter & value \\
\hline \hline$m$ & 23.8 & $Y_{v}$ & -0.8612 \\
$X_{\dot{u}}$ & -2.0 & $Y_{|r| \vartheta}$ & 0.0 \\
$I_{z}$ & 1.76 & $Y_{|u| u}$ & -36.2823 \\
$Y_{\dot{\vartheta}}$ & -10.0 & $Y_{|\vartheta| r}$ & 0.0 \\
$x_{g}$ & 0.046 & $Y_{r}$ & 0.1079 \\
$Y_{\dot{r}}$ & -0.0 & $Y_{|r| r}$ & 0.0 \\
$X_{u}$ & -0.7225 & $N_{\vartheta}$ & 0.1052 \\
$N_{\dot{\vartheta}}$ & 0.0 & $N_{r}$ & 0.0 \\
$X_{|u| u}$ & -1.3274 & $N_{|\vartheta| \vartheta}$ & 5.0437 \\
$N_{\dot{r}}$ & -1.0 & $N_{|r| r}$ & 0.0 \\
$X_{u u u}$ & -5.8664 & $N_{|\vartheta| r}$ & 0.0 \\
$l_{y 1}$ & 0.25 & $l_{y 2}$ & -0.25 \\
$l_{x 1}$ & -0.25 & $l_{x 2}$ & -0.25 \\
$l_{x 3}$ & 1 & & \\
\hline \multicolumn{4}{|r}{}
\end{tabular}

$\operatorname{craft}_{1}$ with $\psi_{1}(0)=0, q_{2}(0)=|1 \mathrm{~m} \quad 20 \mathrm{~m}|^{T}$ is the initial position of $\mathrm{craft}_{2}$ and its orientation is $\psi_{2}(0)=$ $\pi / 2, q_{3}(0)=\left[\begin{array}{ll}15 \mathrm{~m} & 11 \mathrm{~m}\end{array}\right]^{T}$ is the position of the motionless craft 3 . The position of the targets are $\operatorname{Targ}_{1}=$ $\left[\begin{array}{ll}25 \mathrm{~m} & 18 \mathrm{~m}\end{array}\right]^{T}$ and $\operatorname{Targ}_{2}=\left[\begin{array}{ll}25 \mathrm{~m} & 1 \mathrm{~m}\end{array}\right]^{T}$. The radii of different discs used in this simulation are chosen as $r_{1}=r_{2}=r_{3}=2 \mathrm{~m}, \delta_{1}=\delta_{2}=\delta_{3}=1.5 \mathrm{~m}$.

Figure 2 shows the initial position and orientation of two vessels and the third vessel as a stationary obstacle. Figure 3 illustrates the tracking performance of moving vessels to their respective targets in the case of the absence of close obstacles.

Figure 4 shows the navigation performances in the mid-path. In this case, obstacles were detected and the proposed algorithm was able to generate a collision free path $q_{\text {ref } i}$. These graphs clearly show how overlapping between different discs was avoided. Figure 5 depicts the movement of the crafts to their targets in the absence of obstacles.

Figure 6 shows the path followed by the marine

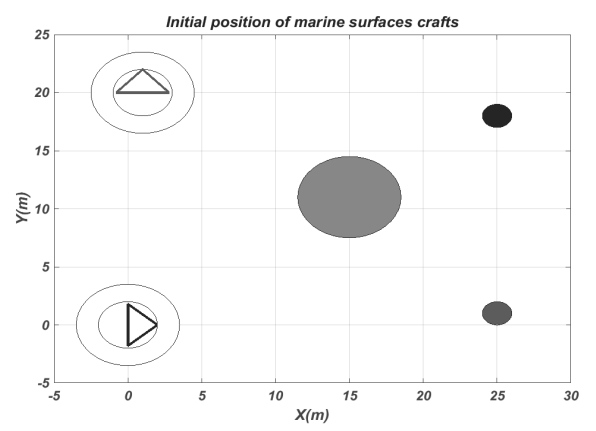

Fig. 2. Initial positions. surface vehicles from the departure points to the arrival targets. The two vessels have reached their targets without colliding with the static vessel nor with each other. Consequently, the proposed on-line algorithm was able to avoid detected dynamic and static obstacles.

Figure 7illustrates the variation in different distances between discs, where marine crafts are immersed. All distances are non-negative. This figure clearly shows that neither collision nor overlapping between different disks occurred during the navigation. Consequently, the trajectory planned by the proposed algorithm ensures an on-line free collision path planning from the initial position to the target.

Figures 8 and 9 display the deduced torques, applied to the marine vessels, computed from the solution of the convex optimization problem. Note that using an Intel i7 desktop PC-8GB RAM, the time average of the solution of the loop is around $3.5 \times 10^{-5} \mathrm{~s}$.

Figure 10 shows the generated forces and the azimuth angles of craft $_{1}$ computed from the solution of the non-convex optimization problem (7) using torques shown in Figs. 8 and 9. It is observed that all control forces are inside the saturation limits. Therefore, the computed forces are feasible and can be applied to $\mathrm{craft}_{1}$. For the azimuth angles, to avoid some unfeasible solutions, a saturation function has been added to keep all solutions inside the desired region. In this simulation, the forces are saturated within the interval $-2 N \leq f \leq 2 N$ and the azimuth angles within the interval $-35^{\circ} \leq \theta \leq 35^{\circ}$.

\section{Case 2. (No static obstacle)}

The second scenario consists in navigation of four ships without static obstacles. The initial configuration is

$$
q^{0}=\left[\begin{array}{cccc}
0 & 0 & 0 & 30 \\
0 & 30 & 15 & 15
\end{array}\right]
$$

It is desired to move these crafts to the desired position

$$
q_{\operatorname{targ}}=\left[\begin{array}{cccc}
30 & 30 & 30 & 0 \\
30 & 0 & 15 & 15
\end{array}\right] .
$$

Using these initial and final configurations, all ships will meet together at the mid-path. The algorithm has to find an optimal direction for each craft to navigate safely to the target. Figure 11 shows the beginning of the navigation of all ships, from their initial positions steering to the targets. Figure 12 illustrates the configuration of all USVs at the mid-path. Figure 13 clearly shows how the proposed algorithm was able to find an optimal direction for each USV in order to avoid the collision with other surface vehicles. Figure 14 depicts the final configuration of all surface vehicles reaching their targets with the generated paths.

It is to be noticed that the proposed algorithm can be used when COLREGs is not respected or in the case of a 


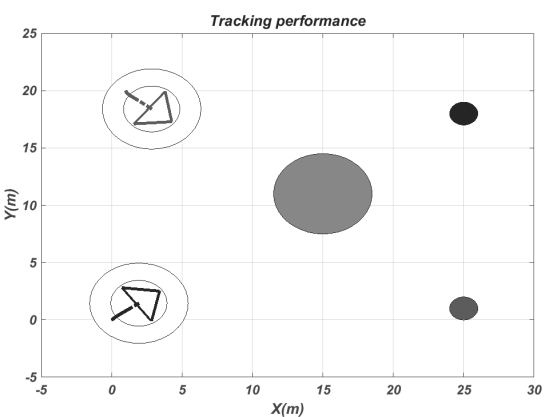

(a)

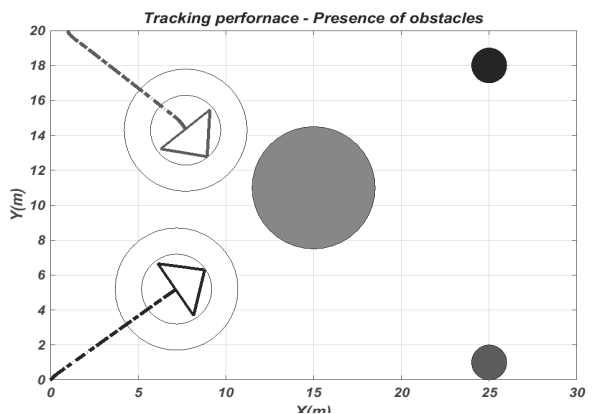

(b)

Fig. 3. Marine vehicle navigation in the absence of obstacles.

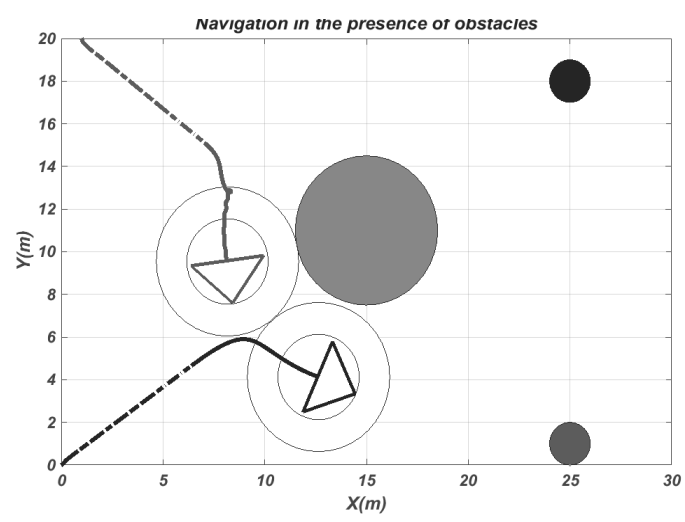

(a)

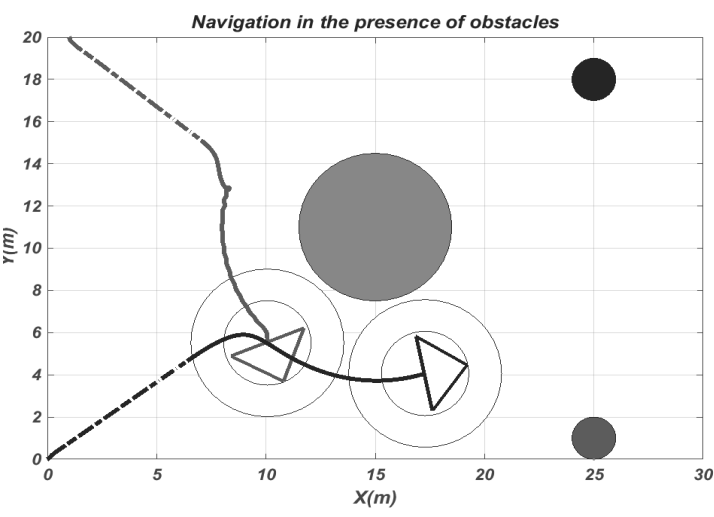

(b)

Fig. 4. Marine vehicle navigation in the presence of obstacles.

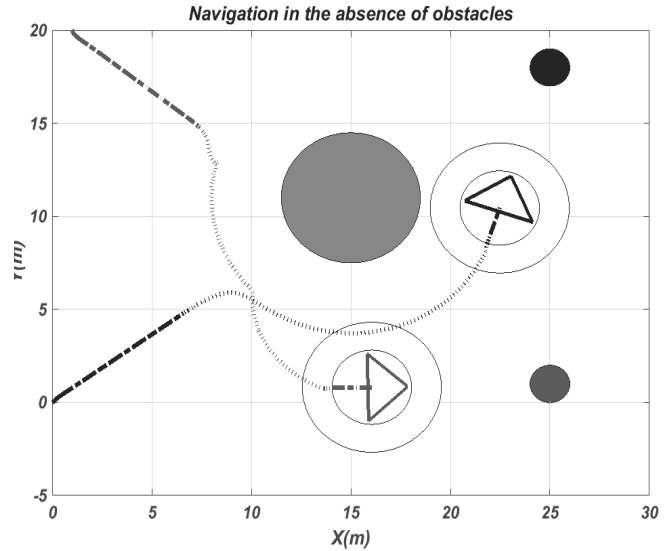

Fig. 5. Marine vehicle navigation in the absence of obstacles.

hazardous environment with low visibility and/or crowded marine traffic. Sensors measure the distance to the nearest obstacle and, whenever this distance is less than a threshold, the emergency obstacle avoidance algorithm

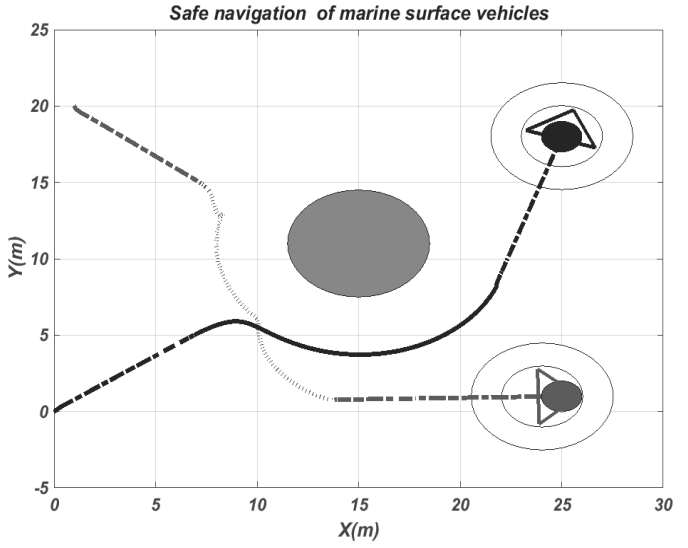

Fig. 6. Planned path to the targets without collision.

imperatively is enabled since the crew cannot navigate the vessel safely. Consequently, the implementation of the proposed algorithm needs on-board advanced electronic equipment (sensors, digital signal processors, etc.). 


\section{Conclusion}

In this paper, an algorithm for planning a collision-free path for multiple autonomous surface vehicles is proposed. The planned via-point trajectory is determined
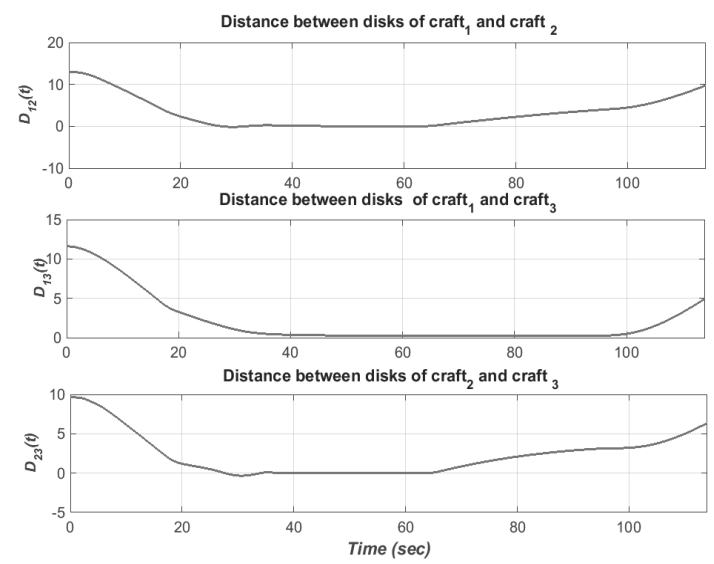

Fig. 7. Distance between different discs.

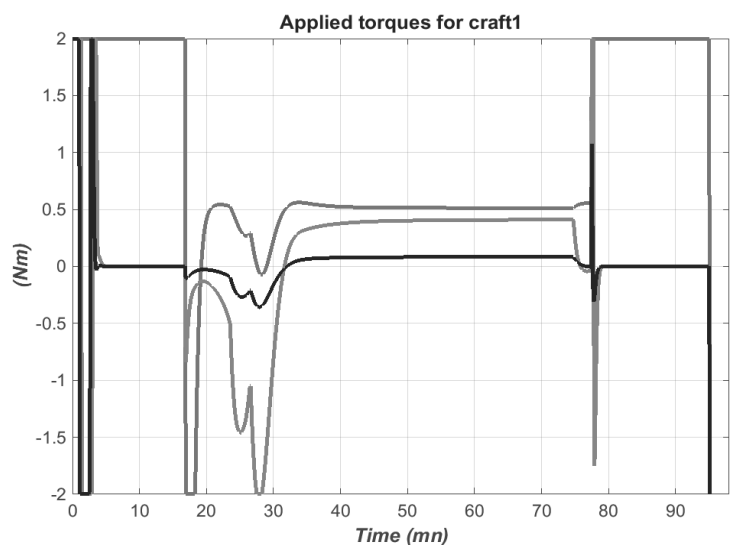

Fig. 8. Applied torques: $\mathrm{Craft}_{1}$.

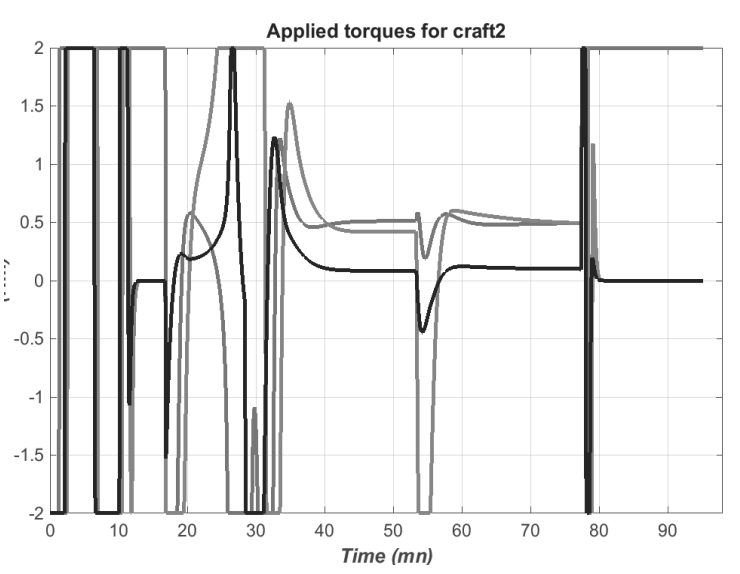

Fig. 9. Applied torques: $\mathrm{Craft}_{2}$. by solving a constrained convex optimization problem. The algorithm is combined with the PD-Feedback linearization controller to navigate the autonomous surface vehicle to its desired target without colliding with both dynamic and static obstacles. A control allocation approach was utilized to minimize the control efforts subject to constrained actuator forces and azimuth angles. The simulation results demonstrate the effectiveness of this algorithm. Hence, by providing necessary sensors and equipment on board, the use of this algorithm will increase the safety and reduce the financial loss that affects the marine sector. The advantages of the proposed algorithm, compared with some approaches cited in the introduction, can be summarized in terms of simplicity in its implementation, low time consumption, and the convergence of the solution to the global minimum is ensured.

Additional research should be oriented firstly to include more general shapes of obstacles, and secondly, towards the real-time implementation of the discrete time algorithm on real vessels.

\section{Acknowledgment}

The authors wish th express their gratitude to the Deanship of Scientific Research at King Saud University for funding the work through the research group project no. RGP-024.

\section{References}

Benjamin, M. Curcio, J. and Newman, P. (2006). Navigation of unmanned marine vehicles in accordance with the rules of the road, Proceedings of the IEEE International Conference on Robotics and Automation, Orlando, FL, USA, Vol. 70, pp. 3581-3587.

Campbell, S. Naeem, W. and Irwin, G.W. (2002). A review on improving the autonomy of unmanned surface vehicles through intelligent collision avoidance maneuvers, Annual Reviews in Control 36(2): 267-283.

Erol, S. Demir, M.E.B. and Eyüboğlu, E. (2018). Analysis of ship accidents in the Istanbul strait using neuro-fuzzy and genetically optimized fuzzy classifiers, The Journal of Navigation 71(2): 419-436.

Fossen, T.I. and Johansen, T.A. (2006). A survey of control allocation methods for ships and underwater vehicles, Proceedings of the 14th Mediterranean Conference on Control and Automation, Ancona, Italy, pp. 1-6.

Fossen, T.L. (1994). Guidance and Control of Ocean Vehicles, Wiley, New York, NY.

Isidori, A. (1989). Nonlinear Control Systems, Springer, London.

Johansen, T.A. and Fossen, T. (2013). Control allocation: A survey, Automatica 49(5): 1087-1103.

Kim, D. Hirayama, K. and Okimoto, T. (2017). Distributed stochastic search algorithm for multi-ship encounter situations, The Journal of Navigation 70(4): 699-718. 


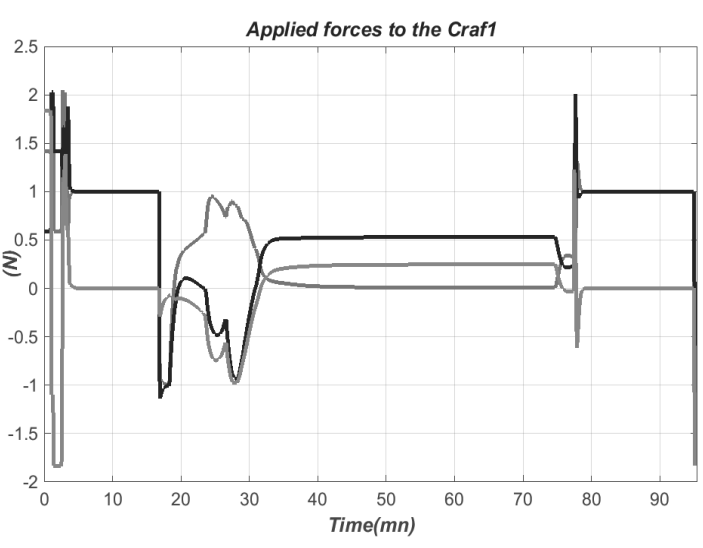

(a)

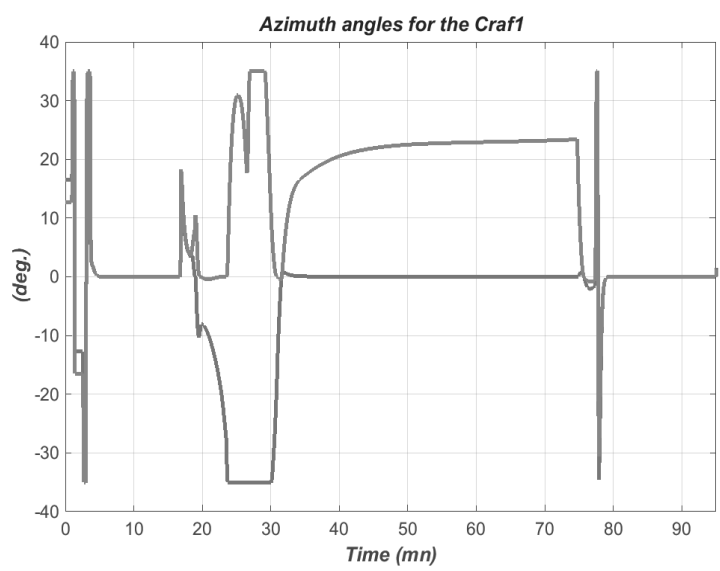

(b)

Fig. 10. Control signals from the control allocation method: forces (a), azimuth angles (b).

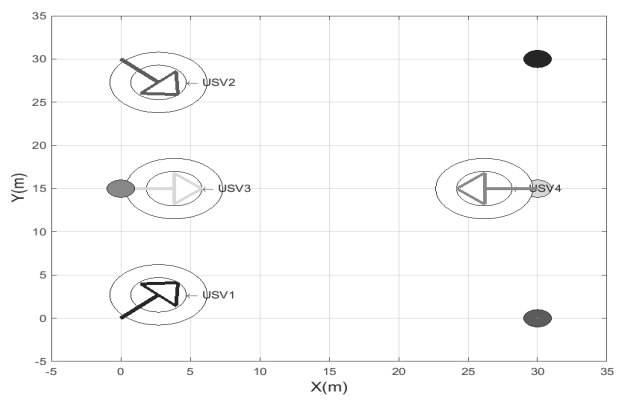

Fig. 11. Beginning of navigation.

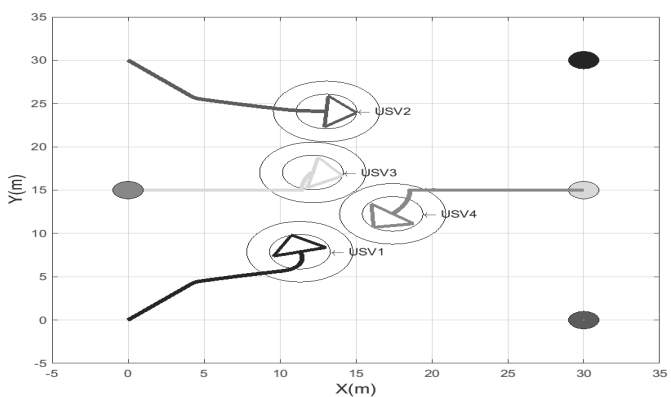

Fig. 12. Navigation performances at the mid-path.

Kuwata, Y., Wolf, M.T.Z.D. and Huntsberger, T.L. (2014). Safe maritime autonomous navigation with COLREGS using velocity obstacles, IEEE Journal of Oceanic Engineering 39(1): 110-119.

Kvasov, D.E. and Mukhametzhanov, M. (2018). Metaheuristic vs. deterministic global optimization algorithms: The univariate case, Applied Mathematics and Computation 318(1): 245-259.

Lazarowska, A. (2015). Ships trajectory planning for collision avoidance at sea based on ant colony optimization, The

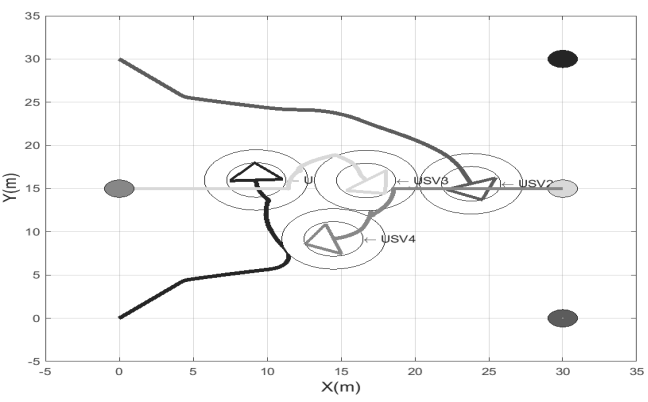

Fig. 13. Obstacle avoidance by USVs.

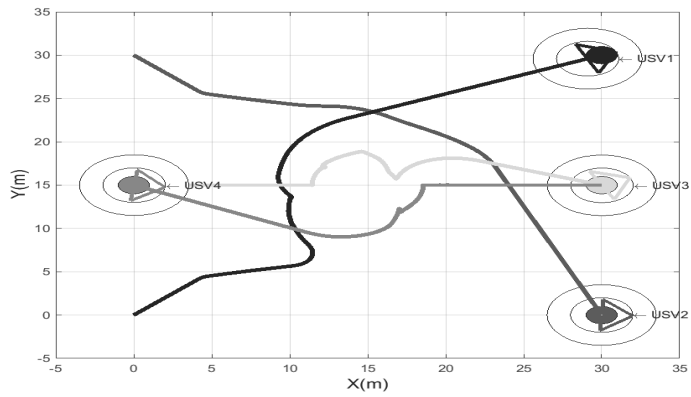

Fig. 14. Final configuration of USVs.

Journal of Navigation 68(2): 291-307.

Liu, Y.H. and Shi, C.J. (2005). A fuzzy-neural inference network for ship collision avoidance, Proceedings of the International Conference on Machine Learning and Cybernetics, Guangzhou, China, pp. 4754-4759.

Mattingley, J. and Boyd, S. (2010). Real-time convex optimization in signal processing: Recent advances that make it easier to design and implement algorithms, IEEE Signal Processing Magazine 27(3): 35-49.

Perera, L.P. Carvalho, J.P. and Soares, C.G. (2011). Fuzzy logic 
based decision-making system for collision avoidance of ocean navigation under critical collision conditions, Journal of Marine Science and Technology 6(1): 84-99.

Skjetne, R. Fossen, T.I. and Kokotovic, P.V. (2005). Adaptive maneuvering, with experiments, for a model ship in marine control laboratory, Automatica 41(2): 289-298.

Statheros, T. Howells, G. and Maier, K.M. (2008). Autonomous ship collision avoidance navigation concepts, technologies and techniques, The Journal of Navigation 61(1): 129-142.

Wang, T. F. Yan, X.P. and Wang, Y. (2017). Ship domain model for multi-ship collision avoidance decision making with colregs based on artificial potential field, International Journal on Maritime Navigation and Safety of Sea Transportation 11(1): 85-92.

$\mathrm{Xu}$, Q. Zhang, C. and Wang, N. (2014). Multi-objective optimization based vessel collision avoidance strategy optimization, Mathematical Problems in Engineering 2014: 1-9.

Zhang, L. Lin, S., Zhou, J. and Papavassiliou, C. (2017). Three-dimensional underwater path planning based on modified wolf pack algorithm, IEEE Access 5: 22783-22795.
Ramdane Hedjar received the $\mathrm{BSc}$ and $\mathrm{PhD}$ degrees from the University of Science and Technology - Houari Boumediene, Algiers, Algeria, in 1988 and 2002, respectively, and the MSc degree from the University of Blida in Algeria in 1992 in electronic and electrical engineering. After obtaining the $\mathrm{PhD}$ degree, he joined the Computer Engineering Department at King Saud University as an assistant professor. From 1992 to 2000, he was a lecturer with the Electronics Department at Djelfa University, and from 1994 to 2000 he was a research assistant with the Electronic Department at the University of Blida. Currently, he is a professor at King Saud University. His research interests include robust control, nonlinear predictive control, robotics, neural network control, and networked control systems.

Messaoud Bounkhel received the BSc degree from the University of Batha, Algeria, in 1994, the MSc degree from the University of Montpellier 2, France, in 1995, and the PhD from the same university in 1999 (all in mathematics). From 1999 to 2001, he was a researcher-lecturer in the Centre of Mathematical Modelling, Santiago, Chile. He joined the Department of Mathematics in KSU, Saudi Arabia, in 2001, where was promoted to a full professor in 2008. His research interests include nonsmooth analysis, mathematical modeling, optimization, differential inclusions, variational inequalities, optimal control theory and applications.

Received: 23 May 2018

Revised: 4 February 2019

Re-revised: 3 June 2019

Accepted: 5 July 2019 